\title{
Guest editorial: special issue on data management in peer-to-peer systems
}

\author{
Beng Chin Ooi · Kian-Lee Tan
}

Published online: 24 September 2009

(C) Springer Science+Business Media, LLC 2009

It has been almost a decade since the Peer-to-Peer (P2P) technology first emerged as a promising paradigm for distributed computing. Over the years, we have witnessed a large number of domain specific P2P systems being utilized. For example, BitTorrent and Gnutella enable users to share any digital files (e.g., music files, video, images); ICQ facilitates exchanges of personal messages; SETI@ home makes computing cycles of participants available; and LOCKSS pools storage resources to archive document collections. Many data structures and overlays that support resource discovery have also been developed. These include CHORD, CAN, BATON and P-Grid.

However, successful deployment is largely associated with sharing of files and resources; most of these P2P systems lack data management capabilities that are typically found in DBMS. The key challenges arise from the scale (large number of peers which far exceeds what traditional distributed databases can handle), the dynamism of the systems (peers can join and leave the network any time), and the lack of a global schema.

For this special issue, we invited original and high-quality submissions addressing all aspects of Data Management in Peer-to-peer Systems. Each submission was reviewed by at least two experts in the field. Due to limited space, we could only accept and select seven papers for this special issue. These papers are summarized as follows.

The first paper Efficient Range Query Processing in Metric Spaces over Highly Distributed Data by C. Doulkeridis, A. Vlachou, Y. Kotidis and M. Vazirgiannis

\footnotetext{
B.C. Ooi · K.-L. Tan $(\bowtie)$

Department of Computer Science, School of Computing, National University of Singapore,

13 Computing Drive, Singapore 117417, Singapore

e-mail: tankl@comp.nus.edu.sg

B.C. Ooi

e-mail: ooibc@comp.nus.edu.sg
} 
presents a framework SIMPEER to address the problem of efficient processing of range queries in metric spaces in a super-peer based P2P network. SIMPEER dynamically clusters peer data and builds distributed routing indices at superpeer level, and evaluates range and $\mathrm{kNN}$ queries of high-dimensional data in a distributed manner. In addition, a recall-based query scheme is proposed to handle result sets of large cardinality. This feature reduces the cost of retrieving the whole answer set while keeping the accuracy of partially retrieved results to be under control.

In the second paper entitled Rewiring Strategies for Semantic Overlay Networks, P. Raftopoulou, E. Petrakis and C. Tryfonopoulos studied how connections between peers in unstructured $\mathrm{P} 2 \mathrm{P}$ systems can be established to improve retrieval effectiveness. By periodically re-clustering peers that are semantically, thematically or socially close into groups, a semantic overlay network can be established. In such a network, links are made between similar peers, while connections between dissimilar peers can be disregarded. The paper gives a comprehensive study of rewiring strategies based on gradient walk and random walk as well as accompanying variants of updating strategies for the routing tables used (short-range and long-range links).

The third paper is entitled Linking Identical Neighborly Partitions for Efficient High-dimensional Similarity Search in Unstructured Peer-to-Peer Systems. The authors, B. Cui, L. Xu and J. Zhao, nicely demonstrated how space partitioning and query routing techniques can be integrated to provide a working solution to process various kinds of data oriented queries, primarily range search and $\mathrm{kNN}$. In the proposed scheme, LINP, each peer summarizes its local data using the space partitioning technique, and exchanges the summarized index with its neighboring peers to construct routing indices. The paper also presents how each peer can reconfigure its neighboring peers to keep relevant peers nearby.

The next paper Maintenance Strategies for Routing Indexes by K. Hose, C. Lemke and K. Sattler investigates the important subject of maintaining the freshness of routing indexes (specifically, data summaries for numerical data) in unstructured P2P networks without global information. It studies several categories of update strategiesin update-driven propagation schemes, updates are propagated through the network either immediately when they occur or after sometime when a specific criterion is fulfilled; in query-driven strategies, updates are triggered either by query receiver node if it realizes that the estimated number of query results is much different from the actual number of query results or by query issuer node using the received results of the query; in update propagation on demand techniques, updates are only executed by update requests that are triggered by peers at predefined interval time.

M. Zhou, W. Qian, X. Gong and A. Zhou studied in their paper Multi-dimensional Data Density Estimation in P2P Networks the important problem of estimating the global data distribution in structured P2P networks. The proposed solution uses a compact multi-dimensional histogram that is distributed across peers in the system. By condensing each local portion by a set of discrete cosine transform coefficients, each peer can hierarchically accumulate the local information to be exchanged with other peers to estimate the global data density.

In the paper DHTJoin: Processing Continuous Join Queries Using DHT Networks, W. Palma, R. Akbarinia, E. Pacitti and P. Valduriez studied the processing of continuous join queries in a distributed data stream system using DHT. The proposed 
method contains two major components: the query dissemination component broadcasts query in the system, and the join processing component exploits indexes to facilitate join processing. It supports two kinds of queries over data streams with time-stamped tuples are supported. The paper also shows that the completeness of the query result is determined by a number of factors including the arrival rate of tuples, the window size of the query, the memory size of nodes, and the number of nodes participating in the join processing.

Finally, the last paper A Cross-Layer Atomic Commit Protocol Implementation for Transaction Processing in Mobile Ad-Hoc Networks by S. Obermeier, S. Böttcher and D. Kleine re-examines the atomic commit problem in the context of mobile adhoc networks. The proposed Cross Layer Commit Protocol (CLCP) is energy efficient, employs all transaction participants as coordinators and uses consensus to deal with failures. Moreover, while it is instantiated from the application layer, it operates on both the network layer and the application layer. The cross layer design enables CLCP to interpret message acknowledgements from message receivers (who sent the acknowledgements) more effectively.

We hope readers will find the content of this special issue interesting and inspiring. We would like to thank all the authors who submitted their papers to this special issue, as well as the many reviewers who have graciously contributed their time and effort to ensure the high quality and timeliness of this special issue. In addition, we thank the two EICs of this journal, A.K. Elmagarmid and A.P. Sheth, for their support and advise. Last, but not least, we are grateful to the staff from Springer, who have been very helpful in finalizing this issue.

Happy reading! 